\title{
Assessment of Ventricular Dissynchrony in Right Ventricular Single Chamber Pacing using Echocardiografic Parameters
}

\author{
Mitre Adriana, Vita I, Dobreanu D \\ Clinic of Cardiology, County Emergency Clinical Hospital, University of Medicine and Pharmacy, Tîrgu Mureș, Romania
}

Background: Right ventricular apical pacing has been used since the early years of pace-makers, despite the fact that it determined nonphysiological ventricular depolarization. As medical technologies developed, septal lead implantation became feasible, in order to outrun the above mentioned inconveniences. The question whether the apical or septal lead position is better still gives rise to a lot of controversies. Different echocardiographic parameters are currently used to assess the impact of specific sites of stimulation on ventricular function. The aim of the study was to determine which of the followed synchronicity parameters varied significantly during apical stimulation, compared to septal stimulation in patients requiring single chamber pacing.

Material and method: Fifty-nine patients admitted between January $1^{\text {st }}$ - December $31^{\text {st }} 2012$ either for battery replacement or for first implant of a single chamber pace-maker were included in this prospective study. Thirty-eight of them had the lead placed in the apex of the right ventricle and 21 on the interventricular septum. All were subjected to echocardiografic examination after device implantation. Measurements included complete chamber and valvular assessment, apart from the synchronicity evaluation, that comprised interventricular mechanical delay (IVMD), septal to posterior wall delay (SPWMD) and electro systolic delays (ESD), in order to assess the presence and compare the relevance of interventricular and intraventricular dissyncrony. All the investigations were in accordance with the Declaration of Helsinki.

Results: Although preejection times were significantly different, there were no statistically significant differences $(p=0.06)$ between the values of IVMD in the two groups, as well as regarding the longitudinal intraventricular dyssynchrony expressed by ESD. Significant differences appeared though, between the values of SPWMD, reflecting the fact that radial intraventricular dissyncrony is present in apical stimulation.

Conclusions: During apical right ventricular pacing, radial intraventricular dissyncrony appears, while in the case of septal pacing this parameter is close to normal. Surprisingly, although right ventricular pacing determines interventricular and longitudinal intraventricular dissincrony, we found no significant differences in this respect, between apical and septal pacing.

Keywords: echocardiography, ventricular dyssinchrony, pacing

Received: 11 June 2013 / Accepted: 31 March 2014

\section{Introduction}

Since the beginning of the pace-maker era, single chamber pacing meant using an apical lead placed in the right ventricle. The internal anatomy of this chamber allows passive lead positioning without special maneuvers. The method is fast, easy to perform and does not require special lead characteristics, despite the fact that it determines nonphysiological ventricular depolarization. By placing the stimulation electrode in the apex of the right ventricle the sequence of depolarization is reversed. The method is still in use, many patients presenting good clinical outcome over decades, and no ventricular functional impairment. On the other hand, some patients develop subsequent systolic dysfunction and this fact raises the suspicion that right ventricular apical pacing might be responsible for the alteration of systolic function in these cases $[1,2]$. As medical technologies developed, septal lead implantation became feasible, in order to outrun the above mentioned inconveniences. The question whether the apical or septal lead position is better, still gives rise to a lot of con-

Correspondence to: Adriana Mitre

E-mail: adimitre@yahoo.com troversies. In both situations QRS complexes get wider in different degrees, the electrical aspect of paced complexes being similar to those of left bundle branch block (LBBB). Although current guidelines define dyssynchrony by QRS duration, there is a poor relation between $L V$ dyssynchrony and QRS width, as one third of patients with QRS $>150$ ms do not show dissynchrony, whereas patients with a narrow QRS often exhibit it [3].

Different methods, including echocardiographic parameters, are currently used to assess the impact of specific sites of stimulation on ventricular function. Various echocardiographic techniques have been proposed to quantify LV dyssynchrony, including simple methods such as Doppler and $\mathrm{M}$-mode echocardiography, or more complex ones, such as pulsed wave and colour tissue Doppler imaging (TDI), deformation imaging, and more recently, three-dimensional echocardiography [4]. Using pulsed wave Doppler recordings across the aortic and pulmonary valves, the aortic and pulmonary pre-ejection times can be determined. An aortic pre-ejection time longer than $140 \mathrm{~ms}$ and a difference between aortic and pulmonary pre-ejection times, also known as interventricular mechanical delay (IVMD), longer than $40 \mathrm{~ms}$ are considered indicative of interven- 
tricular dyssynchrony [5]. Using M-mode in parasternal short or long axis views of the left ventricle, another parameter, septal to posterior wall motion delay (SPWMD), first described by Pitzalis [6], may be obtained, by measuring the time interval between the peak systolic contraction of the septum and the peak inward contraction of the posterior wall. A SPWMD $>130 \mathrm{~ms}$ is a sign of intraventricular radial dyssynchrony [6]. Similar to patients with LBBB, most of the patients with right ventricular pacing present a so called "septal flush" that makes the measurement of SPWMD in M-mode difficult. Therefore, the use of Mmode combined with colour coded tissue Doppler imaging permits the enhancing of wall movements and makes the measurement more reliable. Electrosystolic delays (ESD) are assessed using pulsed wave TDI, by measuring the time interval between QRS onset and peak systolic (S) wave on pulsed-wave-TDI on opposite walls. The presence of intraventricular longitudinal dyssynchrony is defined as a difference between ESD of opposite walls greater than 60 $\mathrm{ms}$, as described by Bax [7].

The aim of this study was to determine which of the followed synchronicity parameters varied significantly during apical stimulation, compared to septal stimulation in patients requiring single chamber pacing.

\section{Material and method}

This prospective study included 62 consecutive patients admitted between January $1^{\text {st }}$ - December $31^{\text {st }} 2012$ to the Cardiology Clinic of the County Emergency Clinical Hospital of Tîrgu Mureș, either for battery replacement or for first implant of a single chamber pace-maker. All patients were subjected to echocardiographic examination after device implantation, using a Vivid S5 GE equipment. Patients with poor acoustic window were excluded, thus pacing parameters were recorded from 59 patients, out of which 38 had the lead placed in the apex of the right ventricle (group I) and 21 on the interventricular septum (group II). The mean age of the patients was $67.8 \pm 8.9$ years for patients with septal stimulation and $69.9 \pm 10$ years for those with apical lead, with a gender ratio of 37/22 males versus females. Measurements included complete chamber and

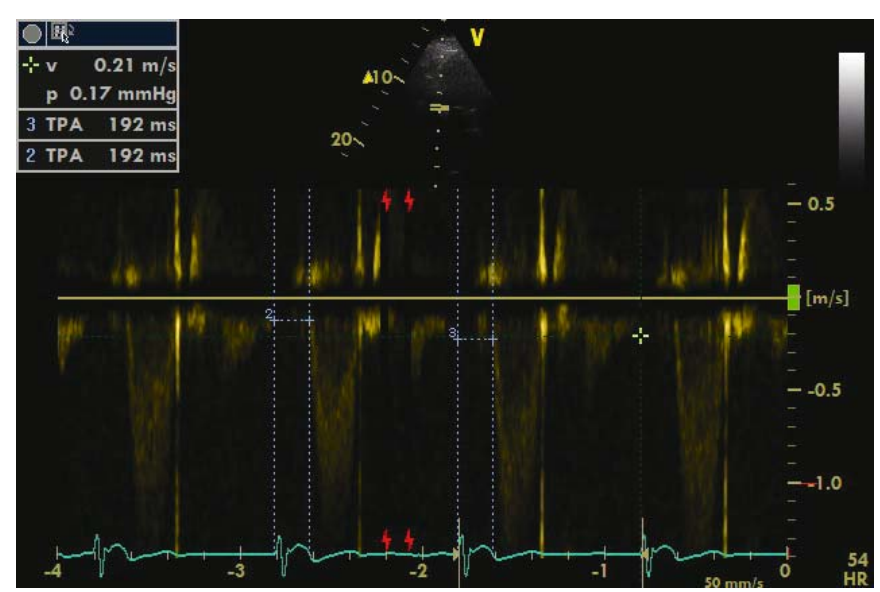

Fig. 1. Method used to measure aortic preejection time
Table I. Mean values for synchronicity parameters measured for the two groups

\begin{tabular}{lcc}
\hline & $\begin{array}{c}\text { Apical stimulation } \\
(\mathrm{n}=38)\end{array}$ & $\begin{array}{c}\text { Septal stimulation } \\
(\mathrm{n}=21)\end{array}$ \\
\hline $\operatorname{TPA}(\mathrm{ms})$ & $149.9( \pm 42)$ & $137.7( \pm 43)$ \\
$\operatorname{TPP}(\mathrm{ms})$ & $115.4( \pm 40)$ & $111.0( \pm 30)$ \\
$\operatorname{IMIV}(\mathrm{ms})$ & $31.8( \pm 2.3)$ & $27.7( \pm 1.2)$ \\
SPWMD $(\mathrm{ms})$ & $118.7( \pm 20.5)$ & $106.2( \pm 12.3)$ \\
ESD1-ESD2 $(\mathrm{ms})$ & $50.9( \pm 2.8)$ & $51.8( \pm 2.3)$ \\
\hline
\end{tabular}

TPA - aortic preejection time; TPP - pulmonary preejection time; IMIV - interventricular mechanical delay; SPWMD - septal to posterior wall motion delay; ESD - electrosystolic delay

valvular assessment, left ventricular functional evaluation, apart from the synchronicity evaluation, that comprised interventricular mechanical delay (IVMD), as a marker of interventricular dyssynchrony, septal to posterior wall motion delay (SPWMD) and electro systolic delays (ESD), as signs of intraventricular dyssynchrony (radial and longitudinal, respectively). Standard echocardiographic measurements were enclosed in our examination protocol for all patients along with dyssynchrony parameters registered in a special chart. Meanwhile, medical history and clinical outcome of the patients were registered. Data obtained were processed using Graphpad.

\section{Results}

Coronary artery disease was the main cause of conduction disturbances that led to pacemaker implantation, in 33 cases $(55.9 \%)$. Other conditions included: dilated cardiomyopathy in 13 cases (22\%), valvular diseases in 12 cases $(20.2 \%)$ and hypertrophic cardiomyopathy, in one patient. Enlarged left ventricle, with an end-diastolic diameter above $60 \mathrm{~mm}$, was found in 17 patients, the majority of them being paced apicaly (12 patients), while 5 patients had a septal lead. A number of 47 patients (77\%) had an ejection fraction above 50\% (16 with septal stimulation and 31 with apical lead). Among the remaining 12 patients, 10 patients (three with septal lead and seven with apical stimulation) had an ejection fraction between 40 $49 \%$ and two were below $40 \%$, both of them with septal lead. Since data regarding the left ventricular systolic func-

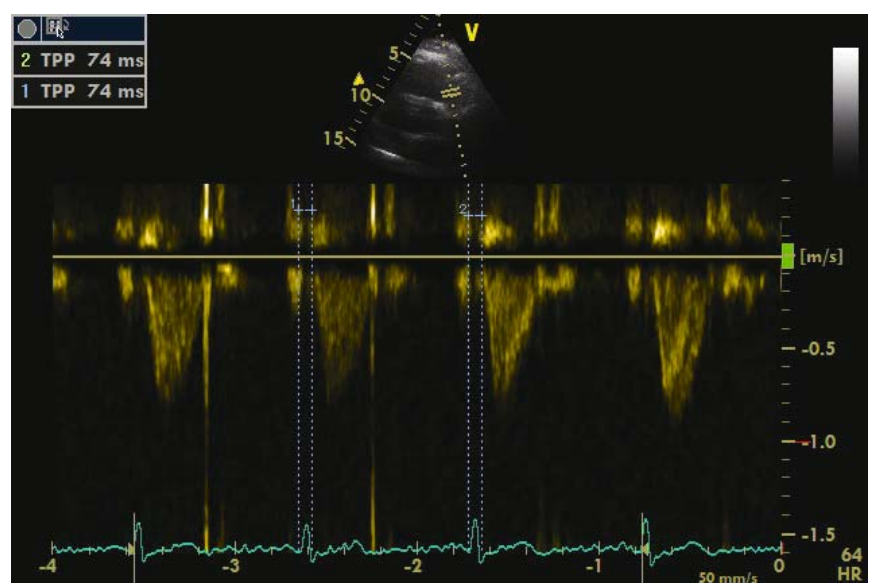

Fig. 2. Method used to measure pulmonary preejection time 


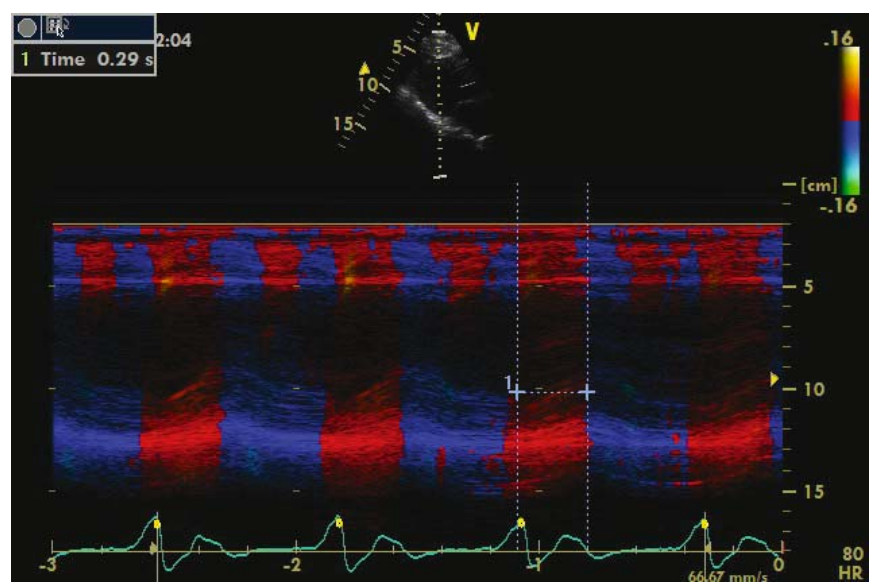

Fig. 3. Method used to determine SPWMD using pulsed wave TDI

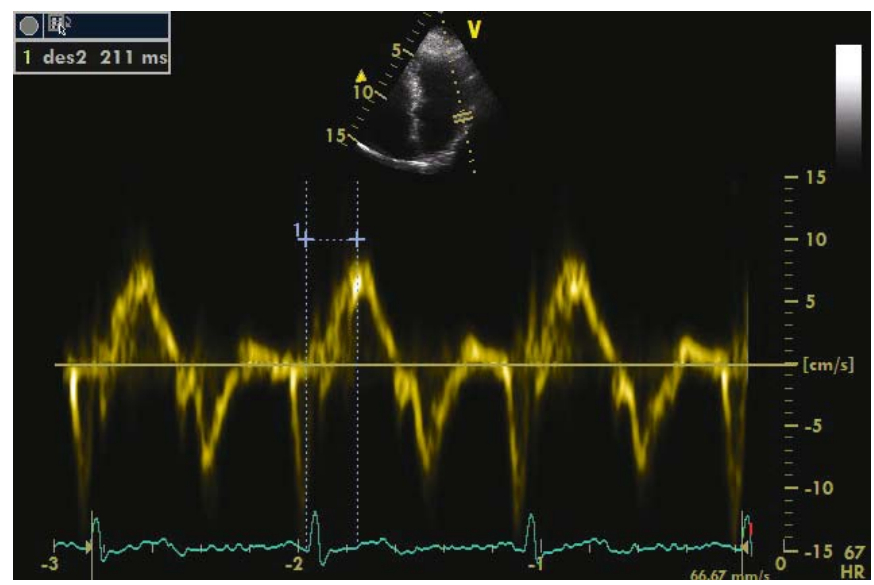

Fig. 5. Measurement of electrosystolic delay at the base of the lateral wall

tion before cardiac pacing were not available in all cases, we were not able to compare these values.

Mean values of preejection times recorded for all 59 patients, as well as mean IVMD for both stimulation regimens and the differences between electrosystolic delays (ESD), are shown in Table I. Depending on the programmed ventricular rate, the preejection times vary, but

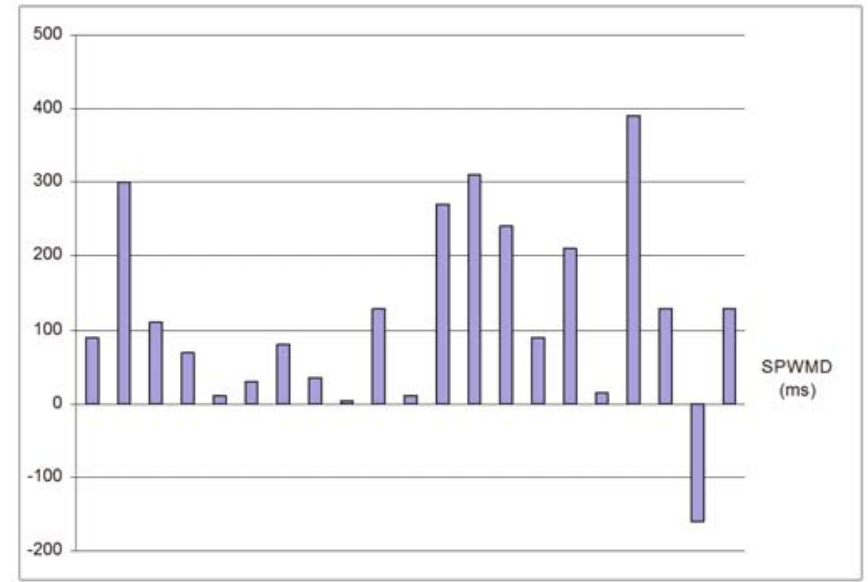

Fig. 6. Values of SPWMD measured for patients with septal stimulation

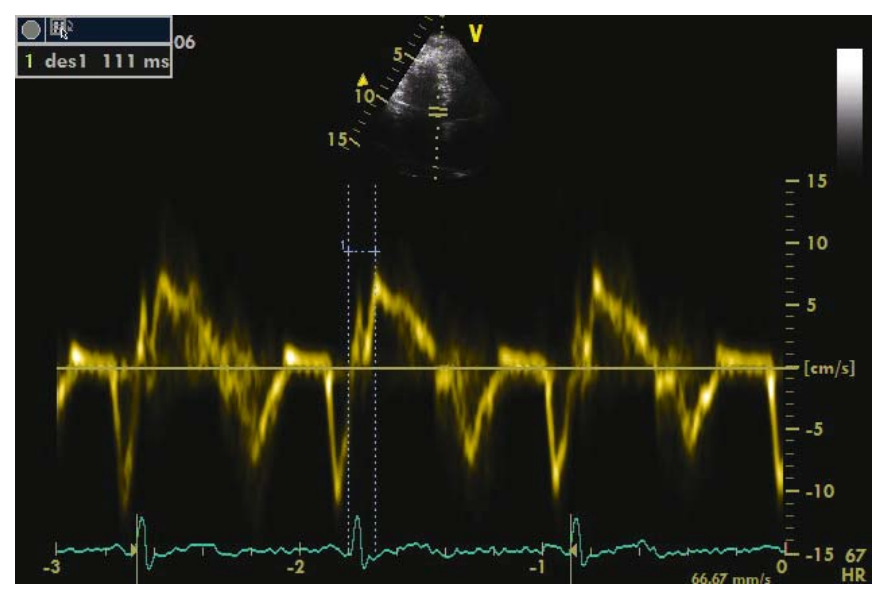

Fig. 4. Measurement of electrosystolic delay at the base of the interventricular septum

the difference between aortic and pulmonary preejection times, IVMD, is not influenced by frequency. Therefore, we compared only the values of IVMD between the two groups. Although preejection times were significantly different, there were no statistically significant differences ( $\mathrm{p}$ $=0.06$ ) between the values of IVMD in the two groups.

Regarding longitudinal intraventricular dyssincrony expressed by the differences between electromechanical delays measured at septal and lateral wall levels, mean values obtained were not consistent statistically with any significance. Significant differences appeared though, between the values of SPWMD. Mean values for patients with septal stimulation (Figure 6) appear to be lower than those for apical pacing (Figure 7), indicating a superior result in case of septal stimulation. However, values of SPWMD varied widely, some of them being collected as negative numbers, and causing a negative skewness.

\section{Discussions}

Following-up pacemaker carriers for years, a decrease in left ventricular systolic function was observed, especially in those having an apical lead [8]. The extent of ventricular functional impairment depends on many factors, that

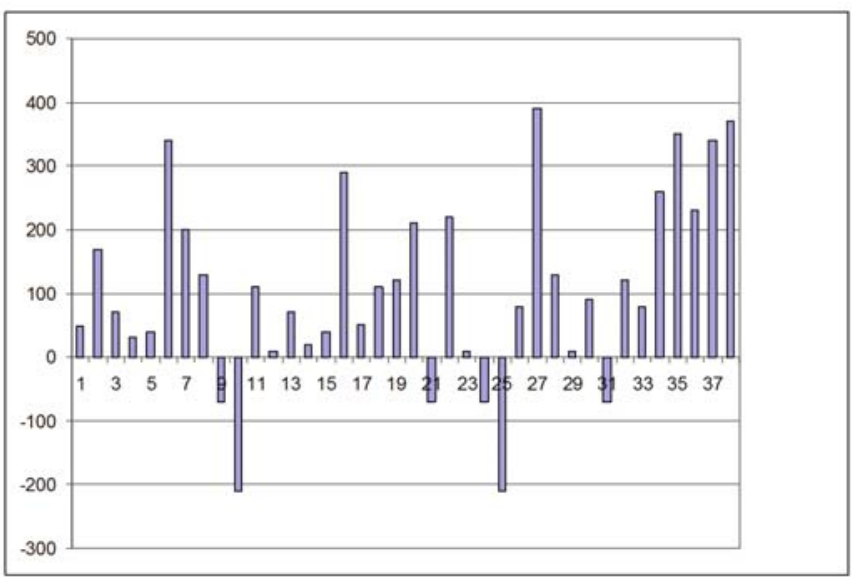

Fig. 7. Values of SPWMD measured for patients with apical stimulation 
include structural changes existent prior to pacing, that are obviously independent of lead position. On the other hand, a discrepancy appeared when patients with no severe left ventricular enlargement developed heart failure after right ventricular pacing. Meanwhile, others experienced no functional impairement. Knowing that during apical stimulation the sequence of ventricular stimulation is altered, this was thought to represent the main cause of new onset heart failure. In order to outrun that, septal pacing was implemented, especially after the results of several clinical trials have been published [9], but controversy still persists. Different methods were used to assess whether septal stimulation is more harmless than apical stimulation. In our study, we found that some echocardiographic parameters can be used in order to point out the changes induced by different sites of stimulation.

Although right ventricular pacing itself induces interventricular dyssynchrony, we found that even if this was present, there was no statistically significant difference between apical and septal pacing in this regard. The main parameter used was the calculation of interventricular mechanical delay (IVMD), by measuring the preejection times $[5,6]$. Another parameter used was the difference of electrosystolic delays (EDS) between two opposite sites of the left ventricle. This parameter is a marker of the existence of intraventricular longitudinal dyssynchrony [10]. In our groups of study, even if the individual values exceeded in some cases the cut-off value of $60 \mathrm{~ms}$, the mean values showed no significant differences. The third echocardiographic parameter that we analyzed was the septal to posterior wall motion delay (SPWMD), as a marker of intraventricular radial dyssynchrony. As it can be seen in Figures 1 and 2, values obtained for this parameter varied widely, some of them being collected as negative numbers, when the time interval between the two structures in motion was highly prolonged. This caused a discrepancy, since the mean values obtained in the statistical analysis were close, but analyzing the medians and the variance we were able to conclude that this was the only parameter that outlined the difference between septal and apical stimulation in favor of the first. This may be responsible in some extent for the decreasing in left ventricular ejection fraction and also for the elevation of left ventricular pressures, possibly following the development of secondary mitral regurgitation.
Similar results were obtained in invasive studies [11], that outlined the fact that the best mechanical performance was achieved when the right ventricle was paced at the interventricular septum in the non-apical mid-to-superior segments. These matters, along with the study of systolic and diastolic function in paced patients were not comprised in our study, but need further extended studies.

\section{Conclusions}

Despite the fact that all patients had interventricular dyssynchrony caused by right ventricular single chamber pacing, only the values of SPWMD were altered in patients with apical pacing compared to those with septal pacing, confirming the fact that intraventricular radial dyssynchrony was associated in case of apical pacing.

\section{References}

1. Nielsen JC, Andersen HR, Thomsen PE, Thuesen L, Mortensen PT, Vesterlund $T$, et al. Heart failure and achocardiographic changes during long-term follow-up of patients with sick sinus syndrome randomized to single-chamber atrail or ventricular pacing. Circulation. 1998;97:987-95.

2. Fang F, Yat-Sun Chan Y, Wai-Kwok Yip G, et al. Prevalence and determinants of left ventricular dyssynchrony in patients with normal ejection fraction received right ventricular apical pacing: a real-time threedimensional echocardiographic study. Eur J Echocardiogr. 2010;11:109118.

3. Bleeker GB, Schalij MJ, Molhoek SG, et al. Relationship between QRS duration and left ventricular dyssynchrony in patients with end stage heart failure. J Cardiovasc Electrophysiol. 2004;15:544-549

4. Ypenburg C, Westenberg JJ, Bleeker GB, et al. Noninvasive imaging in cardiac resynchronization therapy. Pacing Clin Electrophysiol. 2008;31:1475-1499.

5. Leeson P, Mitchel A, Becher $H$ (eds). Echocardiography - handbooks in cardiology, 2nd ed., 2008, Oxford University Press. ISBN 978-0-19921575-1, pg. 204-209

6. Pitzalis MV, Lacoviallo M, Romito $R$, et al. Cardiac resynchronization therapy tailored by echocardiographic evaluation of ventricular asynchrony. J Am Coll Cardiol. 2002;40:1615-1622.

7. Bax JJ, Abraham T, Barold SS, et al. Cardiac resynchronization therapy: part 1- issues before device implantation. J Am Coll Cardiol. 2005;46:2153-2167.

8. Kobza R, Schoenenberger AW, Erne P. Effect of right ventricular pacing on left ventricular ejection fraction in a pacemaker clinic. Acta Cardiol. 2012;67(5):577-82.

9. Weizong W, Zhongshu W, Yujiao Z, et all. Effects of right ventricular nonapical pacing on cardiac function: a meta-analysis of randomized controlled trials. Pacing Clin Electrophysiol. 2013 Feb 25, doi:10.1111/ pace.12112. [Epub ahead of print].

10. Voigt JU. Quantification of left ventricular function and synchrony using tissue Doppler, strain imaging and speckle tracking.Editors: Galiuto E, Badano L, Fox K, et al. The EAE textbook of echocardiography, Oxford, Oxford University Press, 2011, pg. 81-97.

11. Vancura V, Wichterle D, Melenovsky V, KautznerJ. Assessement of optimal right ventricular pacing site using invasive measurements of left ventricular systolic and diastolic function. Europace. 2013;15:1482-1490. 